(2) OPEN ACCESS

\title{
Methotrexate hampers immunogenicity to BNT162b2 mRNA COVID-19 vaccine in immune-mediated inflammatory disease
}

Rebecca H Haberman, ${ }^{1,2}$ Ramin Herati, ${ }^{3,4}$ David Simon (D) , 5arie Samanovic, 3,4 Rebecca B Blank, ${ }^{1,4}$ Michael Tuen, ${ }^{3,4}$ Sergei B Koralov, ${ }^{7}$ Raja Atreya, ${ }^{6,8}$ Koray Tascilar, ${ }^{5,6}$ Joseph R Allen, ${ }^{3}$ Rochelle Castillo, ${ }^{1,2}$ Amber R Cornelius, ${ }^{3}$ Paula Rackoff, ${ }^{1}$ Gary Solomon, ${ }^{1}$ Samrachana Adhikari, ${ }^{9}$ Natalie Azar, ${ }_{1}^{1}$ Pamela Rosenthal, ${ }_{1}^{1}$ Peter Izmirly, ${ }^{1}$ Jonathan Samuels, 1,10 Brian Golden, ${ }^{1}$ Soumya M Reddy, 1,2 Markus F Neurath, ${ }^{6}$ Steven B Abramson (D) , ${ }^{4,11}$ Georg Schett (D) , ${ }^{5,6}$ Mark J Mulligan, 3,4 Jose U Scher (10) 1,2,4

\section{Handling editor Josef $S$ \\ Smolen}

- Additional supplemental material is published online only. To view, please visit the journal online (http://dx.doi. org/10.1136/annrheumdis2021-220597).

For numbered affiliations see end of article.

\section{Correspondence to} Dr Jose U Scher, New York University School of Medicine, New York, NY 10003, USA; jose.scher@nyulangone.org

$\mathrm{RHH}, \mathrm{RH}, \mathrm{DS}$ and MS contributed equally. GS, MJM and JUS contributed equally.

Received 20 April 2021 Accepted 10 May 2021 Published Online First 25 May 2021

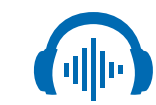

Listen to Podcast ard.bmj.com
Check for updates

(C) Author(s) (or their employer(s)) 2021. Re-use permitted under CC BY-NC. No commercial re-use. See rights and permissions. Published by BMJ.

To cite: Haberman $\mathrm{RH}_{\text {, }}$

Herati $R$, Simon D,

et al. Ann Rheum Dis

2021;80:1339-1344.

\section{ABSTRACT}

Objective To investigate the humoral and cellular immune response to messenger RNA (mRNA) COVID-19 vaccines in patients with immune-mediated inflammatory diseases (IMIDs) on immunomodulatory treatment.

Methods Established patients at New York University Langone Health with IMID $(n=51)$ receiving the BNT162b2 mRNA vaccination were assessed at baseline and after second immunisation. Healthy subjects served as controls $(n=26)$. IgG antibody responses to the spike protein were analysed for humoral response. Cellular immune response to SARS-CoV-2 was further analysed using high-parameter spectral flow cytometry. A second independent, validation cohort of controls $(n=182)$ and patients with IMID ( $n=31$ ) from Erlangen, Germany, were also analysed for humoral immune response.

Results Although healthy subjects $(n=208)$ and patients with IMID on biologic treatments (mostly on tumour necrosis factor blockers, $n=37$ ) demonstrate robust antibody responses (over $90 \%$ ), those patients with IMID on background methotrexate $(n=45)$ achieve an adequate response in only $62.2 \%$ of cases. Similarly, patients with IMID on methotrexate do not demonstrate an increase in CD8+ T-cell activation after vaccination.

Conclusions In two independent cohorts of

patients with IMID, methotrexate, a widely used immunomodulator for the treatment of several IMIDs, adversely affected humoral and cellular immune response to COVID-19 mRNA vaccines. Although precise cut-offs for immunogenicity that correlate with vaccine efficacy are yet to be established, our findings suggest that different strategies may need to be explored in patients with IMID taking methotrexate to increase the chances of immunisation efficacy against SARS-CoV-2 as has been demonstrated for augmenting immunogenicity to other viral vaccines.

\section{INTRODUCTION}

Patients with immune-mediated inflammatory diseases (IMIDs) have an inherently heightened susceptibility to infection and may thus be considered high risk for developing COVID-19. Importantly, however, the strength of response to viral

\section{Key messages}

What is already known about this subject?

- The impact of COVID-19 has been felt across the globe, and new hope has arisen with the approval of messenger RNA (mRNA) vaccines against SARS-CoV-2. Studies have shown immunogenicity and efficacy rates of over $90 \%$ in the immunocompetent adult population. However, there is a lack of knowledge surrounding the response of patients with immune-mediated inflammatory diseases (IMIDs) who may also be on immunomodulatory medications.

- Patients with IMID have been shown to have attenuated immune responses to seasonal influenza vaccination.

What does this study add?

- This study looks at the humoral and cellular immune response to two doses of BNT162b2 mRNA COVID-19 vaccine in participants with IMID (on immunomodulators) compared with healthy controls.

- Individuals with IMID on methotrexate demonstrate up to a $62 \%$ reduced rate of adequate immunogenicity to BNT162b2 mRNA vaccination. Those on anticytokine or nonmethotrexate oral medications demonstrate similar levels of immunogenicity as healthy controls (greater than 90\%).

- Similarly, vaccination did not induce an activated CD8+ T-cell response in participants on background methotrexate, unlike healthy controls and patients with IMID not receiving methotrexate.

vaccines (ie, influenza and hepatitis B) and their long-lasting protective effects in patients with IMID taking conventional disease-modifying antirheumatic drugs (DMARDs), such as methotrexate, or biologic DMARDs, such as tumour necrosis factor inhibitors (TNFis), may not be as robust 


\section{Key messages}

\section{How might this impact on clinical practice or future} developments?

- These results suggest that patients on methotrexate may need alternate vaccination strategies such as additional doses of vaccine, dose modification of methotrexate or even a temporary discontinuation of this drug. Further studies will be required to explore the effect of these approaches on mRNA vaccine immunogenicity.

as it is in the general population following immunisation. ${ }^{1-5}$ Data regarding messenger RNA (mRNA) COVID-19 vaccines' safety, immunogenicity and efficacy are rapidly emerging for the immunocompetent adult population, ${ }^{6}$ where more than $90 \%$ of subjects achieve a satisfactory humoral response. However, the ability of patients with IMID to adequately respond to these vaccines and the differences in humoral and cellular immune response to SARS-CoV-2 vaccination are not known, leaving a significant gap in knowledge that prevents optimal management of this patient population.

Given the experience with seasonal influenza vaccine immunogenicity, ${ }^{27}$ we hypothesised that patients with IMID treated chronically with certain conventional synthetic DMARDs (ie, methotrexate) would have an attenuated response to mRNA COVID-19 vaccines compared with patients with IMID receiving anticytokine treatment or non-IMID participants. To achieve this, we obtained preimmunisation and postimmunisation peripheral blood monocyte cells (PBMCs) and sera from IMID participants $(n=82)$ in two independent cohorts (SAGA (Serologic Testing and Genomic Analysis of Autoimmune, Immune-Mediated and Rheumatic Patients with COVID-19) cohort in New York City, USA, and Erlangen, Germany) and analysed SARS-CoV-2 spike-specific antibody titres compared with non-IMID controls $(n=208)$. Cellular immune responses were further investigated using high-dimensional spectral flow cytometry in the New York City cohort.

\section{METHODS}

\section{Participants}

Established patients with IMID $(n=51)$ receiving methotrexate, anticytokine biologics or both participating in the SAGA study at New York University Langone Health in New York City, ${ }^{8}$ who were receiving BNT162b2 mRNA vaccination were assessed at baseline and after the second dose during the period from 23 December 2020 through 31 March 2021. Healthy subjects served as controls $(n=26)$. IgG antibody responses to the $S$ protein were analysed for humoral immune response. A second independent validation cohort of controls $(n=182)$ and patients with IMID $(n=31)$ on either TNFi or methotrexate monotherapy from Erlangen, Germany, was also analysed for humoral response. Cellular immune responses to the vaccine were also studied for the New York SAGA participants using high-parameter spectral flow cytometry.

\section{Humoral and cellular immune response to BNT162b2 mRNA vaccine}

Humoral immune response was assessed by testing IgG antibodies against the spike protein of SARS-CoV-2. ${ }^{9}$ In the New York City cohort, direct ELISA was used to quantify antibody titres on serum as previously described. ${ }^{10}$ Titre of 5000 units or greater was used as the cut-off to determine an adequate response to vaccination. IgG antibodies against the $\mathrm{S} 1$ domain of the spike protein of SARS-CoV-2 were tested in Erlangen participants using the commercial ELISA from Euroimmun (Lübeck, Germany) on the EUROIMMUN Analyzer I platform and according to the manufacturer's protocol. ${ }^{11}$ Adequate response was defined as greater than $5.7 \mathrm{~nm}$ OD. Immune cell phenotyping before and after immunisation in New York participants was performed by 35-colour spectral flow cytometry on PBMCs. Further details on methodology and analysis can be found in the online supplemental appendix.

\section{Statistical analysis}

Patient characteristics were summarised using means, medians, $\mathrm{SD}$, ranges and percentages as appropriate. $\chi^{2}$ tests of independence and Fisher's exact tests were used for categorical data. Mann-Whitney U and Kruskal-Wallis tests were used for unpaired continuous data, and Wilcoxon signed-rank tests were used for paired continuous data. A p value of less than 0.05 was considered significant. All analyses were done using R V.3.6.0 software (R Foundation for Statistical Computing) and GraphPad Prism V.9 (GraphPad Software).

\section{Patient and public involvement}

This study was designed in response to frequent questions asked by patients with IMID but did not contain any direct public involvement.

\section{RESULTS}

The New York City cohort comprised 26 healthy individuals, 25 individuals with IMID receiving methotrexate monotherapy or in combination with other immunomodulatory medications, and 26 individuals with IMID on anticytokine therapy and/or other oral immunomodulators (table 1). Healthy individuals and those with IMID not on methotrexate were similar in age (49.2 \pm 11.9 years and $49.1 \pm 14.9$ years, respectively), whereas patients with IMID receiving methotrexate were generally older (63.2 \pm 11.9 years). IMID diagnoses were predominantly psoriasis/psoriatic arthritis and rheumatoid arthritis. The Erlangen cohort consisted of 182 healthy subjects, 11 subjects with IMID receiving TNFi monotherapy and 20 subjects with IMID on methotrexate monotherapy (online supplemental table 1). Individuals on methotrexate monotherapy were on average older than healthy individuals and those with IMID not on methotrexate $(54.5 \pm 19.2$ vs $40.8 \pm 12.0$ and $45.0 \pm 15.5$, respectively).

\section{Decreased antibody response to mRNA COVID-19 vaccine in patients with IMID on methotrexate}

Immunogenicity was characterised by testing $\operatorname{IgG}$ antibodies against the spike protein of SARS-CoV-2. In the New York City cohort, of the healthy participants, 25 (96.1\%) of 26 demonstrated adequate humoral immune response. Patients with IMID not on methotrexate achieved a similar rate of high antibody titres $(24 / 26,92.3 \%)$, whereas those on methotrexate had a lower rate of adequate humoral response $(18 / 25,72.0 \%)$ (figure 1A; table 1). This remains true even after the exclusion of patients who had evidence of previous COVID-19 infection $(\mathrm{p}=0.045)$. Median titres were 104354 (range, 141-601185), 113608 (25-737 310) and 46901 (25-694 528) for participants who were healthy, for those with IMID not on methotrexate and for those with IMID on methotrexate, respectively. Similarly, in the Erlangen validation cohort, 179 (98.3\%) of 182 healthy controls, $10(90.9 \%)$ of 11 patients with IMID receiving no methotrexate and $10(50.0 \%)$ of 20 receiving methotrexate 
Table 1 Baseline characteristics and spike-specific SARS-CoV-2 antibody titres in the New York City cohort

\begin{tabular}{|c|c|c|c|c|}
\hline Characteristic & Healthy $(n=26)$ & $\begin{array}{l}\text { IMID } \\
\text { No MTX (n=26) }\end{array}$ & $\begin{array}{l}\text { IMID } \\
\text { Yes MTX }(n=25)\end{array}$ & $P$ value \\
\hline Age, mean (range, SD) & $49.2(28-74,11.9)$ & $49.1(29-79,14.9)$ & $63.2(22-77,11.9)$ & $<0.001$ \\
\hline Female, n (\%) & $16(61.5)$ & $18(69.2)$ & $18(66.7)$ & 0.352 \\
\hline Race, n (\%) & & & & 0.220 \\
\hline White & $16(61.5)$ & $20(76.9)$ & $17(63.0)$ & \\
\hline Black & $1(3.8)$ & $2(7.7)$ & $3(11.1)$ & \\
\hline Asian & $9(34.6)$ & $3(11.5)$ & $3(11.1)$ & \\
\hline Other & $0(0.0)$ & $1(3.8)$ & $2(7.4)$ & \\
\hline Hispanic ethnicity, n (\%) & $1(3.8)$ & $3(11.5)$ & $5(18.5)$ & 0.200 \\
\hline Primary IMID, n (\%) & & & & 0.107 \\
\hline Psoriasis and/or psoriatic arthritis & -- & $15(57.7)$ & $9(36.0)$ & \\
\hline Rheumatoid arthritis & -- & $10(38.5)$ & $12(48.0)$ & \\
\hline Other* & -- & $1(3.8)$ & $4(16.0)$ & \\
\hline \multicolumn{5}{|l|}{ Long-term medication, n (\%) } \\
\hline Methotrexate & -- & $0(0.0)$ & $25(100.0)$ & -- \\
\hline Tumour necrosis factor inhibitor & -- & $11(42.3)$ & $9(36.0)$ & 0.776 \\
\hline Other anticytokines/Janus kinase inhibitors $†$ & -- & $9(34.6)$ & $1(4.0)$ & 0.011 \\
\hline Other oral immunomodulators $\ddagger$ & -- & $7(26.9)$ & $6(24.0)$ & 1.00 \\
\hline Methotrexate dose, mean (SD) & -- & -- & $15.7(5.0)$ & \\
\hline COVID-19 infection before vaccination, $\mathrm{n}(\%)$ & $4(15.4)$ & $5(19.2)$ & $2(8.0)$ & 0.509 \\
\hline Days from first vaccination dose, mean (range, SD) & $29.0(23-44,4.6)$ & $32.5(25-45,5.0)$ & $34.6(21-73,9.9)$ & 0.002 \\
\hline Number receiving second vaccination dose, $\mathrm{n}(\%)$ & $26(100.0)$ & $26(100.0)$ & $25(100.0)$ & 1.00 \\
\hline Adequate humoral response§ף, n (\%) & $25(96.1)$ & $24(92.3)$ & $18(72.0)$ & 0.023 \\
\hline Spike-specific SARS-CoV-2 antibody titres $\uparrow$ & & & & 0.294 \\
\hline Titre median (range) & $104354(141-601$ 185) & $113608(25-737310)$ & 46901 (25-694 528) & \\
\hline
\end{tabular}

*Vasculitis, dermatomyositis, adult-onset Still's disease, sarcoidosis and polymyalgia rheumatica.

tFor IMID No MTX: IL-17i (3), IL-23i (2), abatacept (1), rituximab (1), JAKi (2). For IMID Yes MTX: IL-17 (1).

‡For IMID No MTX: leflunomide (2), oral steroid (1), sulfasalazine (2), apremilast (1), hydroxychloroquine (1). For IMID Yes MTX: oral steroid (2), sulfasalazine (2), hydroxychloroquine (2).

$\S$ Adequate humoral response defined as greater than 5000 units.

ๆAll values 1 week after second vaccination.

IMID, immune-mediated inflammatory disease; MTX, methotrexate.

achieved adequate immunogenicity (figure 1B). Median ODs for this cohort were 9.4 (range, 1.2-14), $7.8(2.3-11.3)$ and 5.9 (0.95-13.5) for participants who were healthy, for those with IMID not on methotrexate and for those with IMID on methotrexate, respectively. Furthermore, when looking at the two cohorts in conjunction ( $\mathrm{n}=290), 204$ (98.1\%) of 208 healthy controls, 34 (91.9\%) of 37 patients with IMID receiving no methotrexate and $28(62.2 \%)$ of 45 receiving methotrexate achieved adequate immunogenicity $(\mathrm{p}<0.001)$ (online supplemental figure S1).

Because of the imbalance in age between groups, we further analysed immunogenicity based on a cut-off age of 55 . In both
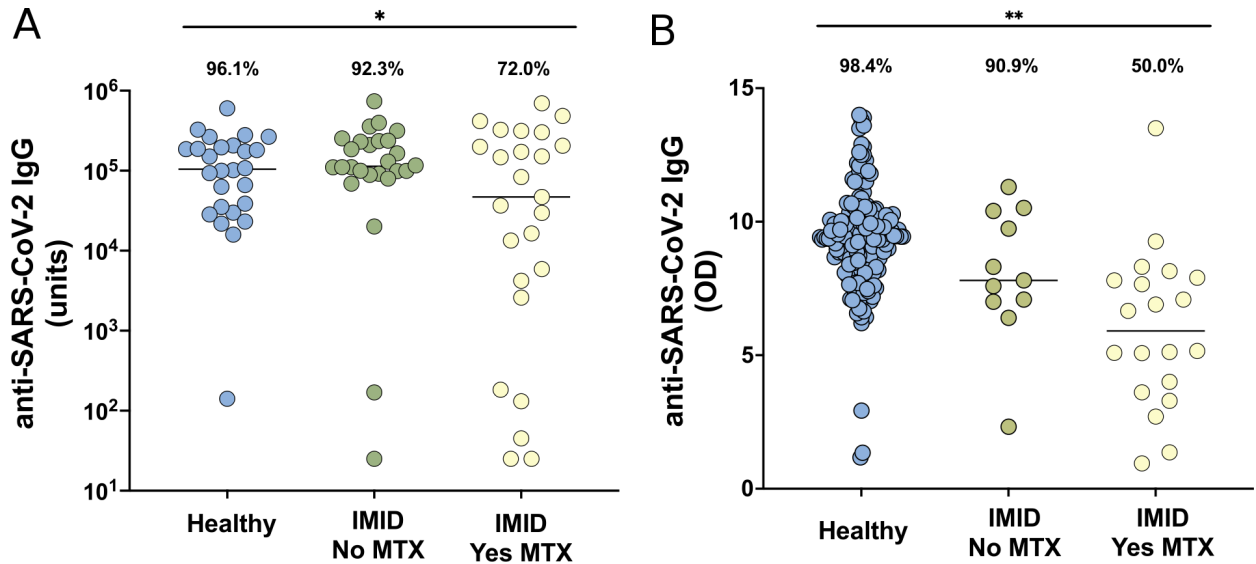

Figure 1 Anti-SARS-CoV-2 IgG levels in cohorts from New York City (A) and Erlangen (B) in healthy participants without IMID (blue), patients with IMID not receiving MTX (green) and patients with IMID treated with MTX (yellow). Solid lines represent mean titre of each group. For the New York City cohort (A), adequate response is defined as greater than 5000 units, and for the Erlangen cohort (B), adequate response is defined as greater than $5.7(O D, 450 \mathrm{~nm}), 2$ SDs of the mean of controls. Percentages and group comparisons using $\chi^{2}$ test of independence reflect proportion of those achieving an adequate response within each group. * indicates $p$ value less than .05 and ${ }^{*}$ indicates $p$ value less than .001 . IMID, immune-mediated inflammatory disease; MTX, methotrexate. 

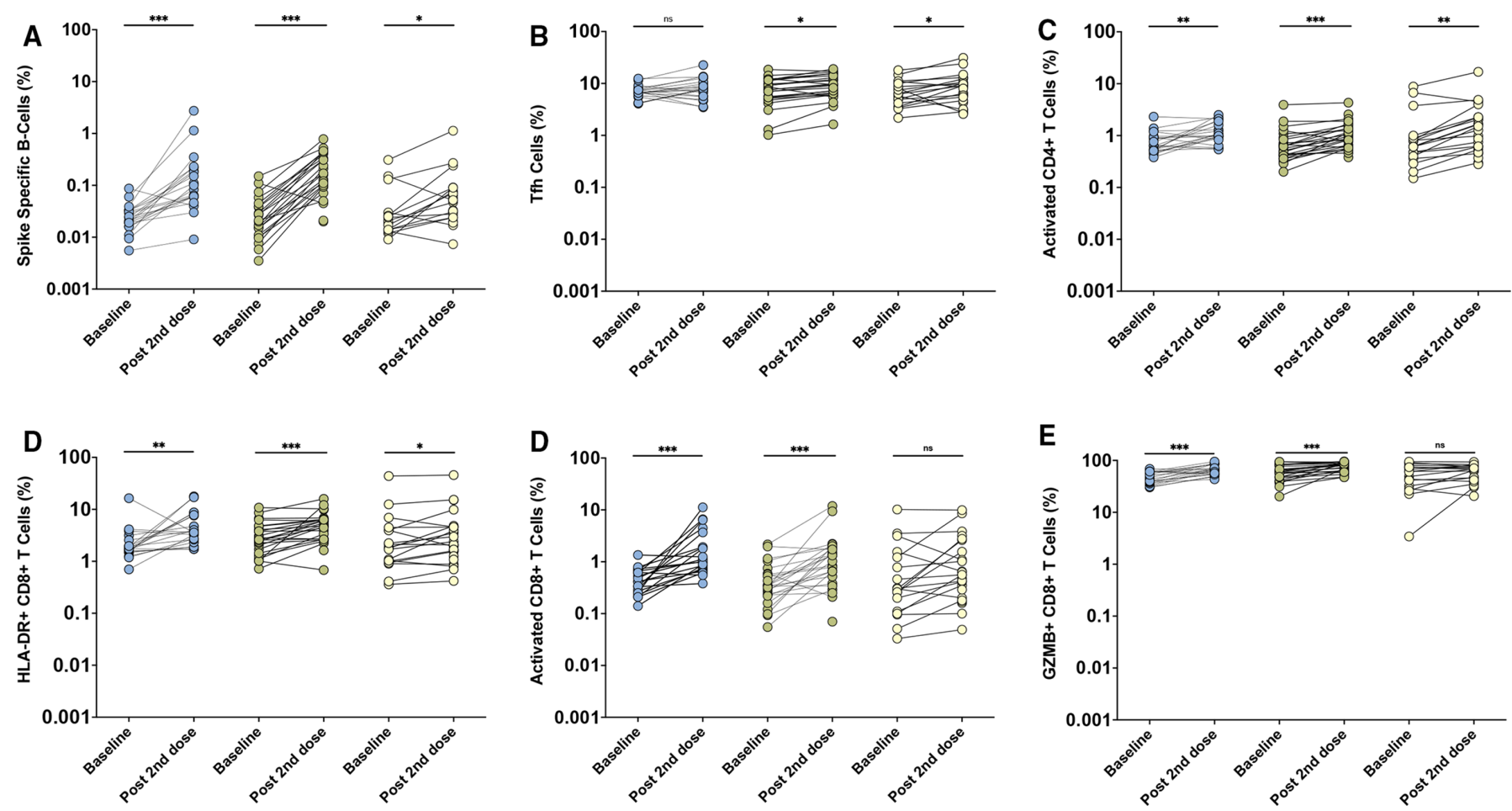

Figure 2 Immune cell populations from the New York City cohort by high spectral flow in healthy controls (blue, $n=20)$, patients with immunemediated inflammatory disease (IMID) not on methotrexate (MTX; green, $n=24$ ) and patients with IMID on MTX (yellow, $n=18$ ), at baseline and after the second dose of BNT162b2 mRNA vaccine. Prevaccination and postvaccination comparisons were performed using Wilcoxon signed-rank tests. Y-axes presented as a logarithmic scale. NS indicates no statistical significance. * indicates $p$ value less than .05. ${ }^{* *}$ indicates $p$ value less than .01. *** indicates $p$ value less than .0001. Tfh, T follicular helper.

age groups, the response rate for those on methotrexate remained significantly lower $(p<0.001)$ (online supplemental figure S2). As an added sensitivity analysis, we used a stricter definition of inadequate antibody response (ie, less than 1000 units for New York City cohort and less than 5 OD for the Erlangen cohort). With the use of these more conservative cut-off levels, patients with IMID on background methotrexate continued to show significantly decreased antibody response $(\mathrm{p}<0.001)$ (online supplemental figure S3).

\section{Lack of CD8+ T-cell activation in patients with IMID on methotrexate following mRNA COVID-19 vaccine}

In the New York City cohort, 20 healthy controls, 24 patients with IMID not receiving methotrexate and 18 patients with IMID who were receiving methotrexate underwent immune cell phenotyping before and after vaccination. The proportions of spike-specific B cells, circulating T follicular helper (cTfh; CD4+ ICOS + CD38 + subset) cells, activated CD4+ T cells and HLA$\mathrm{DR}+\mathrm{CD} 8+\mathrm{T}$ cells increased significantly in all groups after immunisation (figure 2A-D). Activated CD8 + Tcells, defined as CD8 + T cells expressing Ki67 and CD38, and the granzyme B-producing (GZMB) subset of these activated CD8 + Tcells were induced in healthy adults and participants with IMID not on methotrexate, but not induced in patients receiving methotrexate (figure $2 \mathrm{E}, \mathrm{F}$ ).

\section{DISCUSSION}

In two geographically independent cohorts of patients with IMID, we found that methotrexate, a widely used immunomodulator for the treatment of several IMIDs, adversely affected humoral and cellular immunogenicity to COVID-19 mRNA vaccines.
For humoral immunity, the BNT162b2 mRNA vaccines did not induce adequately elevated SARS-CoV-2 spike-specific IgG antibody titres in up to a third of the patients on methotrexate, compared with patients with IMID on other DMARDs, who demonstrated a response as robust as that of healthy controls. This finding was analogous to the previously described effects of methotrexate on influenza vaccine immunogenicity. ${ }^{5}$ 12-14 While a recent report has shown no differences in immunogenicity for patients with IMID, none of the included participants were on methotrexate. ${ }^{15}$ A second study in patients with selfreported rheumatic and musculoskeletal diseases recruited via social media showed that 10 of 13 participants on background methotrexate had detectable antibody levels after only one dose of SARS-CoV-2 mRNA vaccine, ${ }^{16}$ although this was both underpowered and used a semiquantitative ELISA measuring antibodies against SARS-CoV-2 receptor-binding domain. Therefore, the findings from our work looking at antibody responses in patients with IMID after full vaccination regimen are of potentially high clinical relevance because it was recently shown that a temporary discontinuation of methotrexate for 2 weeks significantly improved influenza vaccine immunogenicity in patients with rheumatoid arthritis. ${ }^{2}$

Importantly, the use of high-dimensional spectral flow cytometry allowed for the interrogation of specific cellular immune responses before and after immunisation. Spike-specific B cells, activated CD4 + T cells and cTfh cells were induced similarly in all groups after mRNA vaccination. In contrast, activated CD8+ T-cell responses were notably attenuated in the methotrexate cohort. Moreover, the poor induction of activated CD8 + T cells expressing granzyme $\mathrm{B}$ may indicate reduced cytotoxic functionality of these cells. Indeed, CD8 + T-cell responses were identified to be a correlate of protection in non-human primate studies 
of SARS-CoV-2 infection. ${ }^{17}$ Thus, reduced induction of cytotoxic CD8 + T-cell responses, combined with inconsistent induction of antibody responses, may further impair the effectiveness of COVID-19 vaccines and render patients with IMID on methotrexate more at risk of inadequate vaccine response. However, this finding requires a cautious interpretation as it is quite possible that the use of methotrexate may delay (rather than prevent) adequate cellular mediated immunity against SARS-CoV-2. While spike-specific T-cell immunity has been detected as early as 10 days following one dose of mRNA COVID-19 vaccines in healthy individuals, ${ }^{18}$ mRNA-1273-specific CD4+ and CD8+ T-cell responses were most robustly elicited 2 weeks after the second dose. ${ }^{19}$ Therefore, more detailed and comprehensive studies that include long-term characterisation of the dynamics of cellular responses to these vaccines will be required to understand the clinical implications of these findings.

Although our analysis was limited in sample size, followed participants with biosampling for a relatively short period of time without standardised disease activity status metrics and was restricted to one type of mRNA immunisation, our findings were validated in an independent cohort and revealed that methotrexate, which is widely used for many indications, adversely affected the humoral and cellular immunogenicity to COVID-19 mRNA vaccination. Furthermore, because of the inclusion of patients with prior COVID-19 infection, it is possible that results could be biased in favour of those not on methotrexate. However, when excluding all patients with prior infection, the results remained similar. We also acknowledge that there may have been participants with asymptomatic COVID-19 infection that we have not captured.

While immunosenescence may reduce the level of antibody responses to immunisations, ${ }^{20}$ recent studies on COVID-19 mRNA vaccines have not shown differences in clinical outcomes for the older population. ${ }^{6}$ In our study, patients with IMID on methotrexate were generally older, which may potentially explain some differences in immunogenicity. However, even when looking at participants younger than 55 years, decreased rates of humoral response were still significant. Further validation in even larger cohorts that address efficacy will be required to understand the interaction between age and methotrexate in the context of COVID-19 vaccination.

Importantly, it is not yet clear what level of immunogenicity is representative of vaccine efficacy (and this includes the arbitrary cut-offs chosen for our measurements). We recognise that the definition of adequate cellular and humoral immune response may need to be refined in the future when correlation with efficacy becomes available. However, even after applying more conservative cut-offs, the hampering effects of methotrexate on immunogenicity are still evident.

Taken together, our results suggest that the optimal protection of patients with IMID against COVID-19 will require further studies to determine whether additional doses of vaccine, dose modification of methotrexate or even temporary discontinuation of this drug can boost immune response as has been demonstrated for other viral vaccines in this patient population. ${ }^{7}$

\section{Author affiliations}

'Division of Rheumatology, Department of Medicine, New York University Grossman School of Medicine, New York, New York, USA

${ }^{2}$ NYU Langone Psoriatic Arthritis Center, New York University School of Medicine, New York, New York, USA

${ }^{3}$ Divison of Infectious Disease and Immunology and NYU Langone Vaccine Center, Department of Medicine, New York University School of Medicine, New York, NY, USA

${ }^{4}$ New York University Grossman School of Medicine, New York, New York, USA
}

${ }^{5}$ Department of Internal Medicine 3 - Rheumatology and Immunology, FriedrichAlexander University Erlangen-Nuremberg and Universitätsklinikum Erlangen, Erlangen, Germany

${ }^{6}$ Deutsches Zentrum fuer Immuntherapie (DZI), Friedrich-Alexander University Erlangen-Nuremberg and Universitätsklinikum Erlangen, Erlangen, Germany ${ }^{7}$ Department of Pathology, New York University Grossman School of Medicine, New York, New York, USA

${ }^{8}$ Department of Internal Medicine 1, Friedrich-Alexander University Erlangen-

Nuremberg, Erlangen, Germany

${ }^{9}$ Department of Population Health, NYU Grossman School of Medicine, New York, NY, USA

${ }^{10}$ Department of Medicine, NYU Langone Orthopedic Hospital, New York, New York, USA

${ }^{11}$ Rheumatology Research, NYU-NYU Langone Orthopedic Hospital, New York, New York, USA

Acknowledgements We would like to thank our patients and their families for participating in this study. We are grateful to Luz Alvarado, Rhina Medina, Parvathi Girija, Jyoti Patel and Zakwan Uddin for coordinating and for data entry efforts. We would also like to thank Rebecca Cohen for regulatory support.

Contributors RHH and JUS designed the New York study, designed the data collection tools, analysed and cleaned the data, and drafted and revised the paper. RH, MS and MM designed the New York study, designed and performed the cellular analysis, and revised the paper. SA designed the New York Study and revised the paper. RBB designed the New York study, acquired data and revised the draft. DS, RA, KT, MN and GS designed the Erlangen study, designed the data collection tools, analysed and cleaned the data, and revised the paper. SA aided in original design, statistical analysis and revised the paper. MT, SK, RA and AC analysed data and revised the draft. RC, PR, GS, NA, PR, PI, JS, BG and SMR helped accrue data and revised the draft.

Funding The New York-based studies were funded by NIH/NIAMS (R01AR074500 to JUS; T32-AR-069515 to RHH), NIH/NIAID (UM1Al148574 to MM), Rheumatology Research Foundation (Scientist Development Award to RHH), Bloomberg

Philanthropies COVID-19 Initiative, Pfizer COVID-19 Competitive Grant Programme, The Beatrice Snyder Foundation and The Riley Family Foundation, and the National Psoriasis Foundation. The Erlangen-based studies were supported by the Deutsche Forschungsgemeinschaft (DFG-FOR2886 PANDORA and the CRC1181 Checkpoints for Resolution of Inflammation). Additional funding was received by Bundesministerium für Bildung und Forschung (BMBF; project MASCARA), the Bayerisches Staatsministerium für Wissenschaft und Kunst, the ERC Synergy grant 4D Nanoscope, the IMI funded project RTCure, the Emerging Fields Initiative MIRACLE of the Friedrich-Alexander-Universität Erlangen-Nürnberg and the Else Kröner-Memorial Scholarship (DS, no. 2019_EKMS.27).

Competing interests JUS declares that he has served as a consultant for Janssen, Novartis, Pfizer, Sanofi, UCB and Abbvie and has received funding for investigatorinitiated studies from Novartis, Sanofi and Janssen. GS has served as a consultant for Abbvie, BMS, Eli Lilly, Gilead, GSK Novartis, Janssen and Roche and has received funding for investigator-initiated studies from BMS, Eli Lilly, GSK, Novartis and UCB. MM declares grants from Eli Lilly, Pfizer and Sanofi and personal fees from Meissa Vaccines. PI has received consulting fees from GSK. RHH has received consulting from Janssen. SA reports grant support from Johnson and Johnson. GS declares consulting fees from AbbVie.

\section{Patient consent for publication Not required.}

Ethics approval New York University IRB 20-01078, University Clinic of Erlangen IRB 157_20B.

Provenance and peer review Not commissioned; externally peer reviewed.

Data availability statement All data relevant to the study are included in the article or uploaded as supplementary information. Further deidentified data can be made available upon request. Jose.scher@nyulangone.org.

Twitter David Simon: @DavidSi62640075Mark J. Mulligan: @mjmull

Supplemental material This content has been supplied by the author(s). It has not been vetted by BMJ Publishing Group Limited (BMJ) and may not have been peer-reviewed. Any opinions or recommendations discussed are solely those of the author(s) and are not endorsed by BMJ. BMJ disclaims all liability and responsibility arising from any reliance placed on the content. Where the content includes any translated material, BMJ does not warrant the accuracy and reliability of the translations (including but not limited to local regulations, clinical guidelines, terminology, drug names and drug dosages), and is not responsible for any error and/or omissions arising from translation and adaptation or otherwise.

Open access This is an open access article distributed in accordance with the Creative Commons Attribution Non Commercial (CC BY-NC 4.0) license, which permits others to distribute, remix, adapt, build upon this work non-commercially, and license their derivative works on different terms, provided the original work is 
properly cited, appropriate credit is given, any changes made indicated, and the use is non-commercial. See: http://creativecommons.org/licenses/by-nc/4.0/.

\section{ORCID iDs}

David Simon http://orcid.org/0000-0001-8310-7820

Steven B Abramson http://orcid.org/0000-0002-0668-6344

Georg Schett http://orcid.org/0000-0001-8740-9615

Jose U Scher http://orcid.org/0000-0002-1072-6994

\section{REFERENCES}

1 Caldera F, Hillman L, Saha S, et al. Immunogenicity of high dose influenza vaccine for patients with inflammatory bowel disease on anti-TNF monotherapy: a randomized clinical trial. Inflamm Bowel Dis 2019;142:593-602.

2 Park JK, Lee MA, Lee EY, et al. Effect of methotrexate discontinuation on efficacy of seasonal influenza vaccination in patients with rheumatoid arthritis: a randomised clinical trial. Ann Rheum Dis 2017;76:1559-65.

3 Kobie JJ, Zheng B, Bryk P, et al. Decreased influenza-specific B cell responses in rheumatoid arthritis patients treated with anti-tumor necrosis factor. Arthritis Res The 2011;13:R209.

4 Hagihara Y, Ohfuji S, Watanabe K, et al. Infliximab and/or immunomodulators inhibit immune responses to trivalent influenza vaccination in adults with inflammatory bowel disease. J Crohns Colitis 2014;8:223-33.

5 França ILA, Ribeiro ACM, Aikawa NE, et al. Tnf blockers show distinct patterns of immune response to the pandemic influenza $A$ H1N1 vaccine in inflammatory arthritis patients. Rheumatology 2012;51:2091-8.

6 Polack FP, Thomas SJ, Kitchin N, et al. Safety and efficacy of the BNT162b2 mRNA Covid-19 vaccine. N Engl J Med 2020;383:2603-15.

7 Park JK, Lee YJ, Shin K, et al. Impact of temporary methotrexate discontinuation for 2 weeks on immunogenicity of seasonal influenza vaccination in patients with rheumatoid arthritis: a randomised clinical trial. Ann Rheum Dis 2018;77:898-904.

8 Haberman R, Axelrad J, Chen A, et al. Covid-19 in immune-mediated inflammatory diseases - case series from New York. N Engl J Med 2020;383:85-8.
9 Amanat F, Stadlbauer D, Strohmeier $\mathrm{S}$, et al. A serological assay to detect SARS-CoV-2 seroconversion in humans. Nat Med 2020:26:1033-6.

10 Samanovic MI, Cornelius AR, Wilson JP, et al. Poor antigen-specific responses to the second BNT162b2 mRNA vaccine dose in SARS-CoV-2-experienced individuals. medRxiv 2021:2021.02.07.21251311.

11 Simon D, Tascilar K, Krönke G, et al. Patients with immune-mediated inflammatory diseases receiving cytokine inhibitors have low prevalence of SARS-CoV-2 seroconversion. Nat Commun 2020;11:3774.

12 Ribeiro ACM, Guedes LKN, Moraes JCB, et al. Reduced seroprotection after pandemic H1N1 influenza adjuvant-free vaccination in patients with rheumatoid arthritis: implications for clinical practice. Ann Rheum Dis 2011;70:2144-7.

13 Adler S, Krivine A, Weix J, et al. Protective effect of A/H1N1 vaccination in immunemediated disease--a prospectively controlled vaccination study. Rheumatology 2012;51:695-700

14 Hua C, Barnetche T, Combe B, et al. Effect of methotrexate, anti-tumor necrosis factor $\alpha$, and rituximab on the immune response to influenza and pneumococcal vaccines in patients with rheumatoid arthritis: a systematic review and meta-analysis. Arthritis Care Res 2014;66:1016-26.

15 Geisen UM, Berner DK, Tran F. Immunogenicity and safety of anti-SARS-CoV-2 mRNA vaccines in patients with chronic inflammatory conditions and immunosuppressive therapy in a monocentric cohort. Ann Rheum Dis 2021;80:1302-7.

16 Boyarsky BJ, Ruddy JA, Connolly CM, et al. Antibody response to a single dose of SARS-CoV-2 mRNA vaccine in patients with rheumatic and musculoskeletal diseases. Ann Rheum Dis 2021:80:1098-9.

17 McMahan K, Yu J, Mercado NB, et al. Correlates of protection against SARS-CoV-2 in rhesus macaques. Nature 2021:590:630-4.

18 Kalimuddin S, Tham CY, Qui M, et al. Early T cell and binding antibody responses are associated with Covid-19 RNA vaccine efficacy onset. Med 2021. doi:10.1016/j. medj.2021.04.003. [Epub ahead of print: 08 Apr 2021].

19 Jackson LA, Anderson EJ, Rouphael NG, et al. An mRNA Vaccine against SARS-CoV-2 Preliminary Report. N Engl J Med 2020;383:1920-31.

20 Walsh EE, Frenck RW, Falsey AR, et al. Safety and immunogenicity of two RNA-based Covid-19 vaccine candidates. N Eng/ J Med 2020;383:2439-50. 